\title{
SUR QUELQUES LEPTOMONAS D'EUPHORBES DE MADAGASCAR
}

\author{
Par Odette TUZET et Yvon CABANIS
}

A notre connaissance, l'étude des Flagellés des Euphorbes de Madagascar n'a pas été reprise depuis les travaux déjà anciens de Lafont (1911).

Leptomonas davidi a été rencontré en abondance par Carougeau, puis par Le Fur, dans le latex d'Euphorbia pilulifera et E. thymifolia, à Tamatave, Diégo-Suarez, Majunga, ainsi que dans l'île de Nossi-Bé.

Vincent observe à la Réunion le Leptomonas davidi chez Euphorbia pilulifera, E. thymifolia et E. hypericifolia. A Zanzibar, E. pilulifera est parasité.

Au Congo Belge, Rodhain et Béquéart (1911) signalent des Leptomonas dans le latex d'Euphorbia indica. Dans le Haut-Sénégal et le Niger (Léger, 1911), au Dahomey (Bouet et Roubaud, 1911), Euphorbia pilulifera héberge aussi des Flagellés.

Les Leptomonas des Euphorbes n'ayant pas été observés sur les plateaux environnant Tananarive, un de nous a examiné le latex de sept espèces d'Euphorbes récoltées dans cette région : Euphorbia rosea, E. milii tananarivea, E. milii milii, E. milii breoni, E. enteromorpha, E. pauliani et $E$. durani. Toutes hébergeaient des Flagellés. Des Leptomonas n'avaient été jusqu'à présent signalés dans aucune de ces Euphorbes.

Nous avons trouvé, à côté de Leptomonas de type davidi, des Flagellés montrant avec cette espèce de grandes différences de taille. Nous en avons fait seulement deux nouvelles variétés qui sont, pensons-nous, des variétés écologiques, dues à une composition différente des latex. Nous avons remarqué, en effet, des différences microscopiques importantes dans les latex des diverses espèces d'Euphorbes que nous avons étudiées.

\section{Euphorbia rosea}

Cette Euphorbe montre, dans les exemplaires examinés, des Leptomonas, toujours rares, de l'espèce davidi. Le corps mesure $16 \mu$ de 
long et l'épaisseur faible est d'environ 1,5 $\mu$. Le blépharoplaste est bien visible et le noyau, ovoïde, est logé dans le tiers antérieur du corps (fig. 1).

\section{Euphorbia milii tananarivae}

Les Flagellés sont ici très nombreux. Leur taille, variable, oscille entre 9 et $20 \mu$ de long, le flagelle est généralement moins long que le corps. L'extrémité postérieure est arrondie et l'antérieure plus effilée. Le noyau, situé dans le tiers antérieur du corps, est ovoïde, plus petit que celui du Leptomonas davidi typique et le blépharoplaste est toujours bien visible (fig. 2).

Malgré quelques caractères particuliers de ces parasites, nous ne les séparerons pas du Leptomonas davidi. Il est possible que les différences de forme observées soient dues à la consistance du latex, qui est peut-être différente de celui des Euphorbes hébergeant le $L$. davidi sous sa forme typique.

\section{Euphorbia milii et E. milii breonie}

Ces deux espèces d'Euphorbes montrent des Leptomonas semblables. Ils sont toujours de petite taille, ne dépassant pas $10 \mu$ à 10,5 $\mu$ de long (fig. 3). En dehors de cette différence de taille, leur aspect est le même que celui de Leptomonas davidi. Nous avons observé de nombreuses divisions longitudinales. Migone (1916) a créé une nouvelle espèce, Leptomonas elmassiana, pour un Flagellé de petite taille (14 à $15 \mu$ de long). Mais l'hôte n'est plus une Euphorbe, mais une Asclépiadacée du Brésil. Il nous paraît difficile de rapprocher la petite forme du latex d'E. milii milii et $E$. milii breonie de cette espèce ; nous préférons ne pas la séparer du Leptomonas davidi et en faire la variété minor.

\section{Euphorbia enteromorpha}

Cette Euphorbe héberge des Flagellés nombreux, de plus grande taille que les Leptomonas davidi typiques. Ils peuvent atteindre $23 \mu$ de long avec un Flagellé d'au moins $18 \mu$. Ils sont minces et généralement peu tordus. L'extrémité postérieure est effilée et le blépharoplaste presque terminal (fig. 4). Les différences entre ces Flagellés et $L$. davidi sont trop peu importantes pour que nous les séparions. Elles peuvent être simplement le fait de l'hôte différent. Nous ferons, de ce parasite, une variété major du Leptomonas davidi. 


\section{Euphorbia pauliani}

Les Leptomonas, généralement peu abondants, peuvent atteindre $35 \mu$ de longueur pour le corps et une cinquantaine de microns pour le flagelle (fig. 5). Si l'on en excepte cette taille inhabituelle, leur aspect général est celui du Leptomonas davidi.

\section{Euphorbia durani}

Cette espèce d'Euphorbe possède aussi des Leptomonas de grande taille ; leur corps mesure de 25 à $26 \mu$ de long (fig. 6, $a$ ) ; le flagelle est moins développé que celui du parasite de $E$. pauliani. Les Leptomonas sont abondants et les divisions binaires nombreuses (fig. 6, $b$ ). Ces dernières commencent généralement par la partie postérieure du corps.

Nous avons retrouvé, dans le latex d'Euphorbia durani, des formes montrant une dégénérescence nucléaire par fragmentation du noyau (fig. $6, c$ ). Des images semblables ont d'ailleurs été observées par França (1911) dans les Leptomonas davidi altérés, et par Beaurepaire-Aragao (1927) dans le Phytomonas françai du latex du Maniol palmata du Brésil.

Nous avons aussi observé, comme dans l'espèce précédente, à côté des grandes formes typiques, des formes souvent plus petites, à noyau unique, ou quelquefois fragmenté en deux granules et qui sont toujours plus ou moins enroulés. Mais nous n'avons pas vu leur transformation en formes leishmaniennes.

\section{RÉSUMÉ}

Nous avons observé des Leptomonas dans sept espèces d'Euphorbes récoltées sur les hauts plateaux environnant Tananarive : E. rosea, E. milii tananarivea, E. milii milii, E. milii breonie, E. enteromorpha, E. pauliani et E. durani.

Chez $E$. rosea et $E$. milii tananarivea, les parasites du latex sont des Leptomonas davidi typiques, malgré l'extrémité postérieure généralement arrondie du parasite d'E. milii tananarivea.

Euphorbia milii milii et E. milii breoni hébergent des Leptomonas de petite taille, dont la longueur ne dépasse pas $10 \mu$, la longueur moyenne de $L$. davidi étant de $16,5 \mu$ à $19,5 \mu$; nous proposons de faire de ce Leptomonas une variété du L. davidi : Leptomonas davidi var. minor.

Par contre, Euphorbia enteromorpha, E. pauliani et E. durani montrent des Leptomonas beaucoup plus longs que les $L$. davidi typiques. Leur taille varie de $35 \mu$ de long chez $E$. pauliani à 23 ou 


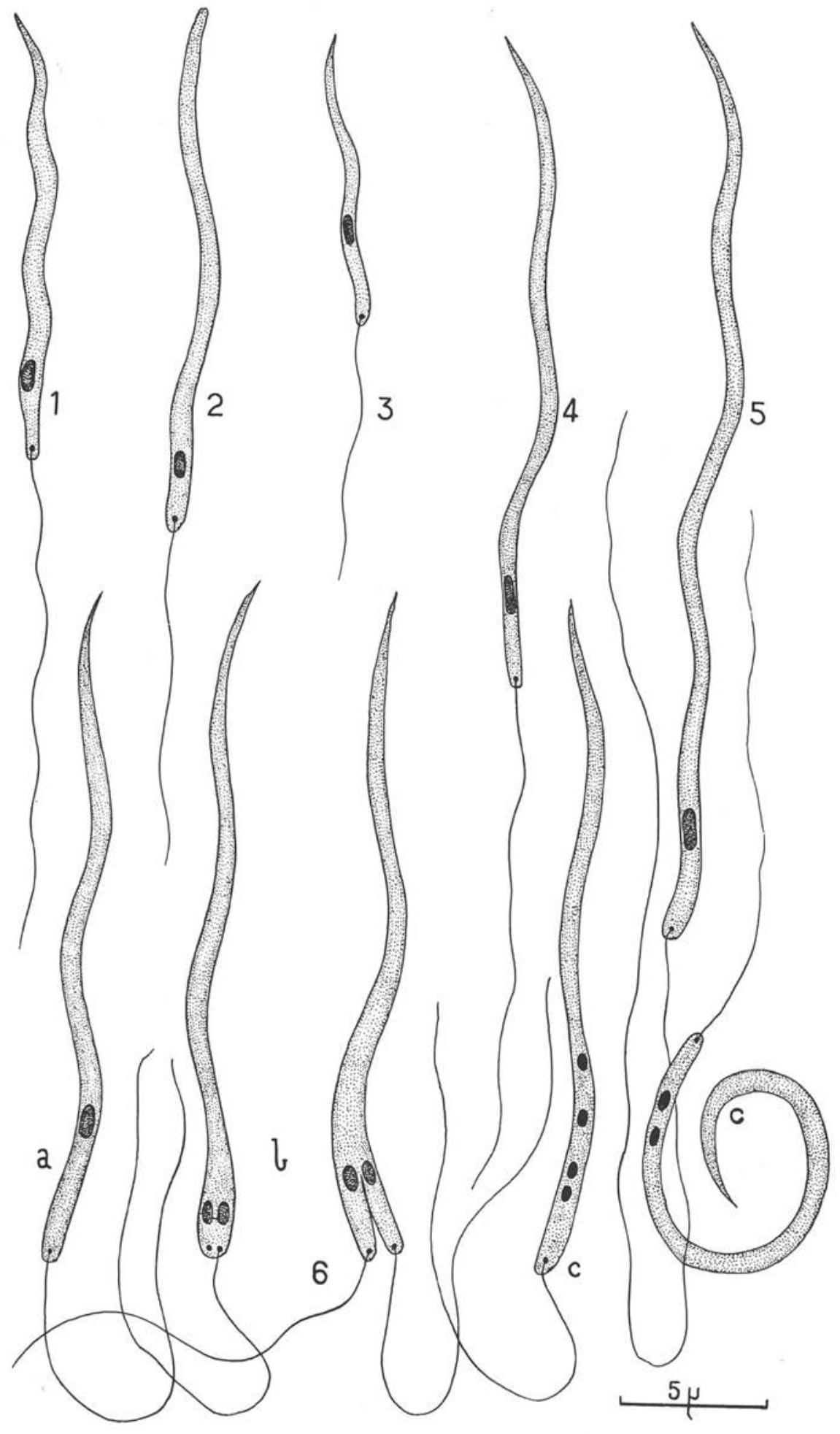


$26 \mu$ chez E. enteromorpha et E. durani. Le parasite d'E. durani nous a montré une dégénérescence nucléaire et de petites formes semblables à celles décrites par Beaurepaire-Aragao chez le Phytomonas françai. Malgré la grande taille de ce Phytomonas (25 à $40 \mu$ de long, avec flagelle de 40 à $60 \mu$ ), nous ne pensons pas, vu l'hôte différent, pouvoir l'identifier avec les Leptomonas d'E. enteromorpha, $E$. pauliani et $E$. durani. Nous ne les identifierons pas non plus au Leptomonas bordasi, décrit par França (1921) chez une Asclépiadacée, Morreiro odorata (sa taille va de 24 à $27 \mu$, mais le flagelle ne mesure que 7,5 à $9 \mu$ ).

Nous ferons des parasites d'E. enteromorpha, E. pauliani et E. durani une variété du Leptomonas davidi : Leptomonas davidi var. major.

\section{Auteurs CITÉs}

Beaurepaire-Argao (H. de), 1927. - Sur un Flagellé du latex de Maniol palmata, Phytomonas françai n. sp. C.R. Soc. Biol., vol. 97, p. 1077-1079.

Bonet (G.) et Roubaud (E.), 1921. - Sur la présence au Dahomey et le mode de transmission de Leptomonas davidi Lafont. Flagellé parasite des Euphorbiacées. C.R. Soc. Biol., vol. 70, pp. 55-57.

França (C.), 1911. - Quelques notes sur Leptomonas davidi Lafont. Bull. Soc. Path. Exot., vol. 4, pp. 669-671.

- 1921. - Sur deux Phytoflagellés ( $L$. elmassiani Migone et $L$. bordasi n. sp.). Ann. Soc. Belge de Médecine Tropicale, vol. 1, pp. 245-254.

Lafont (A.), 1909. - Sur la présence d'un parasite de la classe des Flagellés dans le latex de l'Euphorbia pilulifera. C R. Soc. Biol., vol. 66, p. 1011.

1911. - Observations sur Leptomonas davidi. Bull. Soc. Path. Exot., vol. 4 , pp. 464-467.

LÉGer (A.), 1911. - Présence de Leptomonas davidi Lafont dant l'Euphorbia pilulifera du Haut-Sénégal et Niger. Bull. Soc. Path. Exot., vol. 4, p. 626-627.

Migone (L.-E.), 1916. - Un nouveau Flagellé des plantes : Leptomonas elmassiani. Bull. Soc. Path. Exot., vol. 9, p. 356-359.

Rodhain (J.) et Bequeart (J.), 1911. - Présence de Leptomonas dans le latex d'une Euphorbe congolaise. Bull. Soc. Path. Exot., vol. 4, pp. 198-200.

[Faculté des Sciences de Montpellier (Hérault)]

Fia. 1. - Leptomonas davidi du latex d'Euphorbia rosea

Fig. 2. - Leptomonas davidi du latex d'Euphorbia milii tananarivea

Fiti. 3. - Leptomonas davidi var. minor n. var.

du latex d'Euphorbia milii milii et E. milii breonie

Fig. 4. - Leptomonas davidi var. major

du latex d'Euphorbia enteromorpha n. var.

Fig. 5. - Leptomonas davidi var. major n. var. du latex d'Euphorbia pauliani FIG. 6. - Leptomonas davidi var. major n. var. du latex d'Euphorbia durani $a$, forme normale ; $b$, deux stades de division; $c$, formes de dégénérescence 